\title{
Analysis of common targets for circular RNAs
}

Ya-Chi Lin ${ }^{1,2+}$, Yueh-Chun Lee ${ }^{3,4+}$ (D), Kai-Li Chang ${ }^{5}$ (D) and Kuei-Yang Hsiao ${ }^{6,7,8,9^{*}}$ (D)

\begin{abstract}
Background: The studies of functions of circular RNAs (circRNAs) are heavily focused on the regulation of gene expression through interactions with multiple miRNAs. However, the number of predicted target genes is typically overwhelming due to the synergistic complexity caused by two factors - the binding of multiple miRNAs to a circRNA and the existence of multiple targets for each miRNA. Analysis of common targets (ACT) was designed to facilitate the identification of potential circRNA targets.

Results: We demonstrated the feasibility of the proposed feature/measurement to assess which genes are more likely to be regulated by circRNAs with given sequences by calculating the level of co-regulation by multiple miRNAs. The web service is made freely available at http://lab-x-omics.nchu.edu.tw/ACT_Server.

Conclusions: ACT allows users to identify potential circRNA-regulated genes and their associated pathways for further investigation.
\end{abstract}

Keywords: Circular RNA, microRNA, Common targets, miRNA sponge

\section{Background}

Circular RNA (circRNA) is a newly recognized class of single stranded regulatory RNA molecules with ends covalently closed through a backsplice between a downstream splice donor and an upstream splice acceptor. Recent discoveries through sequencing technology and computational analyses have revealed the widespread existence of circRNAs in animal cells and many other organisms [1-3].

CircRNAs contribute to transcriptional activation, post-transcriptional modulation, translation, and protein interactions [4-8]. Among these, the most popularly studied function of circRNA is that of a miRNA sponge that regulates the gene expression network [9-11]. Pioneer studies have made great contributions dissecting and archiving these relationships among miRNAs, circRNAs, and associated pathological phenotypes [12-15]. However, studies investigating the biological functions of circRNAs are largely limited to the scope of a single

\footnotetext{
* Correspondence: ky.hsiao@nchu.edu.tw

${ }^{\dagger}$ Ya-Chi Lin and Yueh-Chun Lee contributed equally to this work

${ }^{6}$ Institute of Biochemistry, College of Life Sciences, National Chung Hsing

University, Taichung 40227, Taiwan

${ }^{7}$ Program in Translational Medicine, College of Life Sciences, National Chung

Hsing University, Taichung 40227, Taiwan

Full list of author information is available at the end of the article
}

miRNA linked to a single gene [16-18]. Thus, how to identify a manageable gene list length and to consider its role as a whole for further functional characterization has become a critical task.

In this study, we developed and tested an intuitive concept that genes targeted by more circRNAassociated miRNAs are more likely to be modulated by a given circRNA. We established and provided a web service for the analysis of common targets (ACT) for circRNAs to facilitate the molecular characterization of the biological functions of various circRNAs.

\section{Implementation}

The circRNA-associated miRNA-gene network typically involves many genes targeted only once by a miRNA (Additional file 1: Figure S1, gray nodes), and thus these genes may be less efficiently regulated by a given circRNA. The central idea of ACT is to identify target genes with high binding numbers for circRNAassociated miRNAs (Additional file 1: Figure S1, blue nodes). To implement this analysis, miRNA-binding sites in circRNAs were first extracted (Fig. 1a - Step

(c) The Author(s). 2019 Open Access This article is distributed under the terms of the Creative Commons Attribution 4.0 International License (http://creativecommons.org/licenses/by/4.0/), which permits unrestricted use, distribution, and reproduction in any medium, provided you give appropriate credit to the original author(s) and the source, provide a link to the Creative Commons license, and indicate if changes were made. The Creative Commons Public Domain Dedication waiver (http://creativecommons.org/publicdomain/zero/1.0/) applies to the data made available in this article, unless otherwise stated. 
A

(1) Populate the list of miRNAs

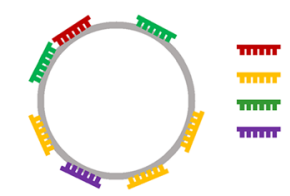

(2) Find targets for each miRNA

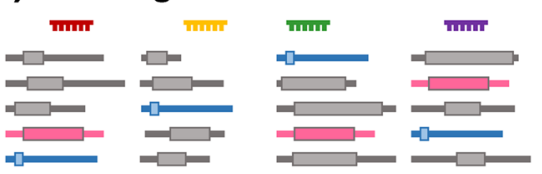

(3) Computing Common Targets

Gene $X$ targeted by circRNA-associated miRNAs $r, y, g$ \&

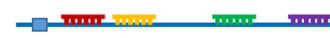

$C T_{\text {gene } X}=\#$ of binding sites $(r+y+g+p)$

$=n b s_{m i R N A_{r}}+n b s_{m i R N A_{y}}+n b s_{m i R N A_{g}}+n b s_{m i R N A_{p}}$
B

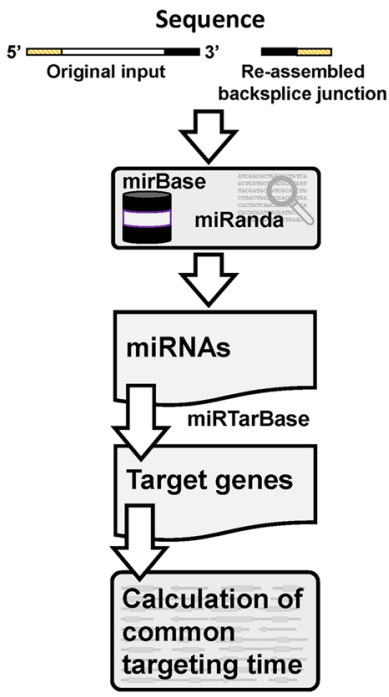

Fig. 1 Schematic illustration of ACT. a In step 1, miRNAs that bind to the given circRNA were identified by the presence of binding sites. In addition, the number of binding sites (nbs) for each miRNA was recorded for further analysis in step 3. In step 2, the targets of each miRNA were identified. It should be noted that some genes (colored in blue and pink) were targets of multiple circRNA-associated miRNAs. In step 3, the nbs for miRNAs that bind to the same gene were summed and used for further sorting. $\mathbf{b}$ The databases and tools integrated in ACT (see the section on implementation)

1), followed by the identification of target genes for each circRNA-associated miRNA (Fig. 1 - Step 2). Finally, the targeting number for each gene was calculated (Fig. 1 - Step 3). The flowchart for the sequent processes is summarized in Fig. 1b. The ACT server takes circular RNA sequences in FASTA format. The default miRNA sequences are downloaded from miRBase (Release 22) [19, 20]. First, the user-inputted sequence is extracted from the $5^{\prime}$ and $3^{\prime}$ ends (30 nt) and reverse-joined to produce a backsplice junction. Then the list of miRNAs that potentially bind to the given circRNA is generated by miRanda software (version 3.3a, Aug 2010) [21] using mature miRNA sequences from miRBase. In order to reduce the number of predicted miRNA binding sites, the parameter '-strict' is applied when using miRanda. The position of miRNAs spanning the backsplice junction is calibrated to the beginning of the original inputted sequence, and the number of miRNA binding sites $(n b s)$ is recorded for further calculations. A list of the target genes for each miRNA with binding site(s) on the given circRNA is generated using miRTarBase (Release 7.0) [22]. The gene list is then collapsed for unique entries, and for each gene $(g)$, the number of targets for circRNA-associated miRNAs (from the last step) is calculated as the sum of $n b s$ (see Fig. 1a Step 3 for example). The genes in the list are ranked by the common targeting time (CT).
Common Targeting time of Gene $g\left(C T_{g}\right)=\sum_{m i R N A s \in g} n b s$

\section{Results}

ACT-selected genes are enriched in specific biological pathways

ACT performs a distinct assessment compared to other metrics that measure the binding energy or pairing score between miRNA and circRNA. Compared to the density of miRNA binding sites, the binding energy and pairing score given by miRanda for these predicted miRNAs on circRNAs, CT for genes provides a more dynamic range to distinguish circRNAs with/without identified miRNA sponge activity and a background dataset (Additional file 1: Figure S2A-D and Table S1). The web interface for ACT is neat and the output files are annotated with detailed information (Fig. 2a and b). CircHIPK3, a previously identified circRNA that targets multiple miRNAs [10], was used as an example (provided for users in the web interface, Fig. 2a). Raw analysis using only the miRNA-target relationship revealed 7350 genes, and approximately half of these genes were targeted by one or two miRNAs (Fig. 3a and b; 3842 out of 7350, 52.27\%). ACT is aimed at identifying common targets that are targeted by several circRNA-associated miRNAs. The top 100 genes were exported and are listed in Fig. 3a. A few 


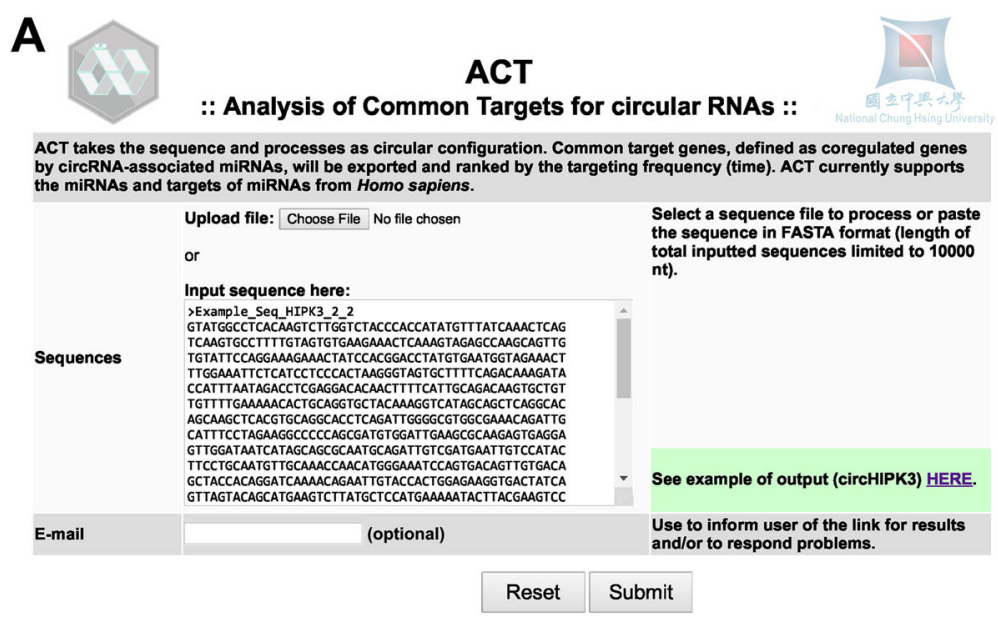

B

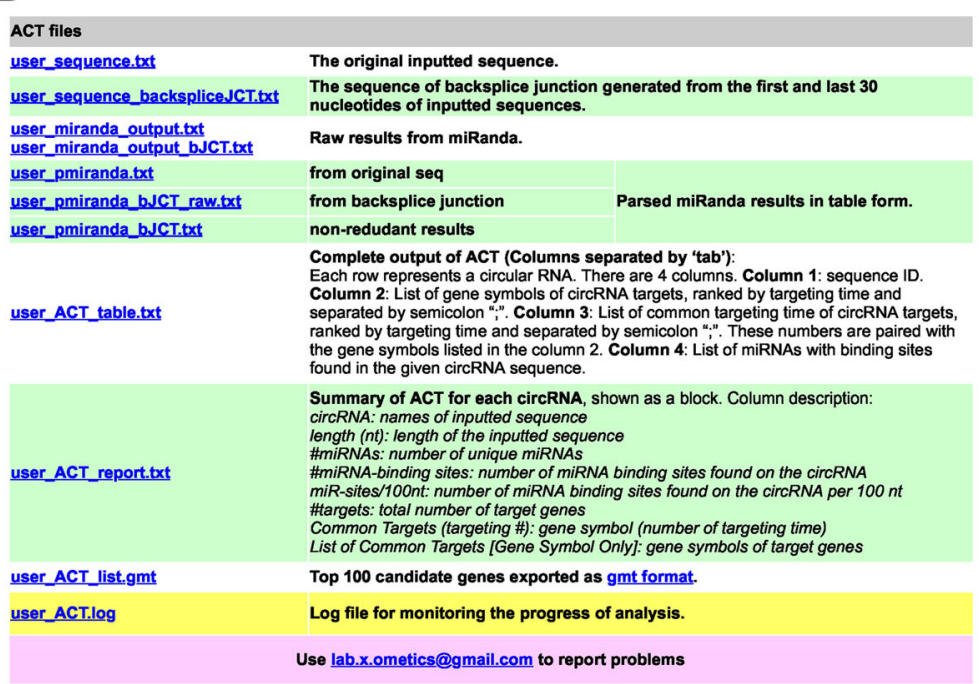

Fig. 2 Web interface for ACT. a The start page provides a simple and straightforward interface for users to input the necessary information. An example sequence and a link for an example of the analysis are provided (circHIPK3). b The analysis example using circHIPK3 is provided with detailed annotation

known circRNAs with and without known sponge activity to multiple miRNAs were used for comparison. Cytoplasmic circRNAs including circHIPK3, circCCDC66, circPVT1 and circIRAK3 [11, 23, 24], previously reported to function as molecular sponges for multiple miRNAs, and nuclear circRNAs from FLI1 and UBR5 genes with distinct molecular functions other than as miRNA sponges in the nuclei $[25,26]$ were applied to the ACT pipeline. To characterize whether the ACT-predicted circRNA-regulated genes play biological roles, we adapted the concept of co-regulation or the convergence of regulation. We assumed that the circRNA-targeted genes are more likely to be conserved and involved in the same pathways during evolution. The ACT-selected genes were subjected to pathway enrichment analysis. The results of pathway enrichment analysis of the genes selected by ACT from these cytoplasmic circRNAs with miRNA sponge activity demonstrated that these genes tended to be enriched or clustered in the same pathways (Fig. 3c). In sharp contrast, the ACT-selected genes from two nuclear circRNAs (either top- or bottom-ranked ones) showed no pathway enrichment (gene lists provided in Additional file 1: Table S2). The lack of convergence in the regulation of the pathways implied that the molecular functions of these nuclear circRNAs were less likely to be as regulators than as miRNA sponges.

\section{ACT enables the distinguishment of circRNAs with or without potential miRNA sponge activity}

To further elucidate the performance and potential application of ACT, we evaluated the convergence of pathway regulation among different metric-derived gene lists. The target genes of the top $10 \%$ of miRNAs according to the 

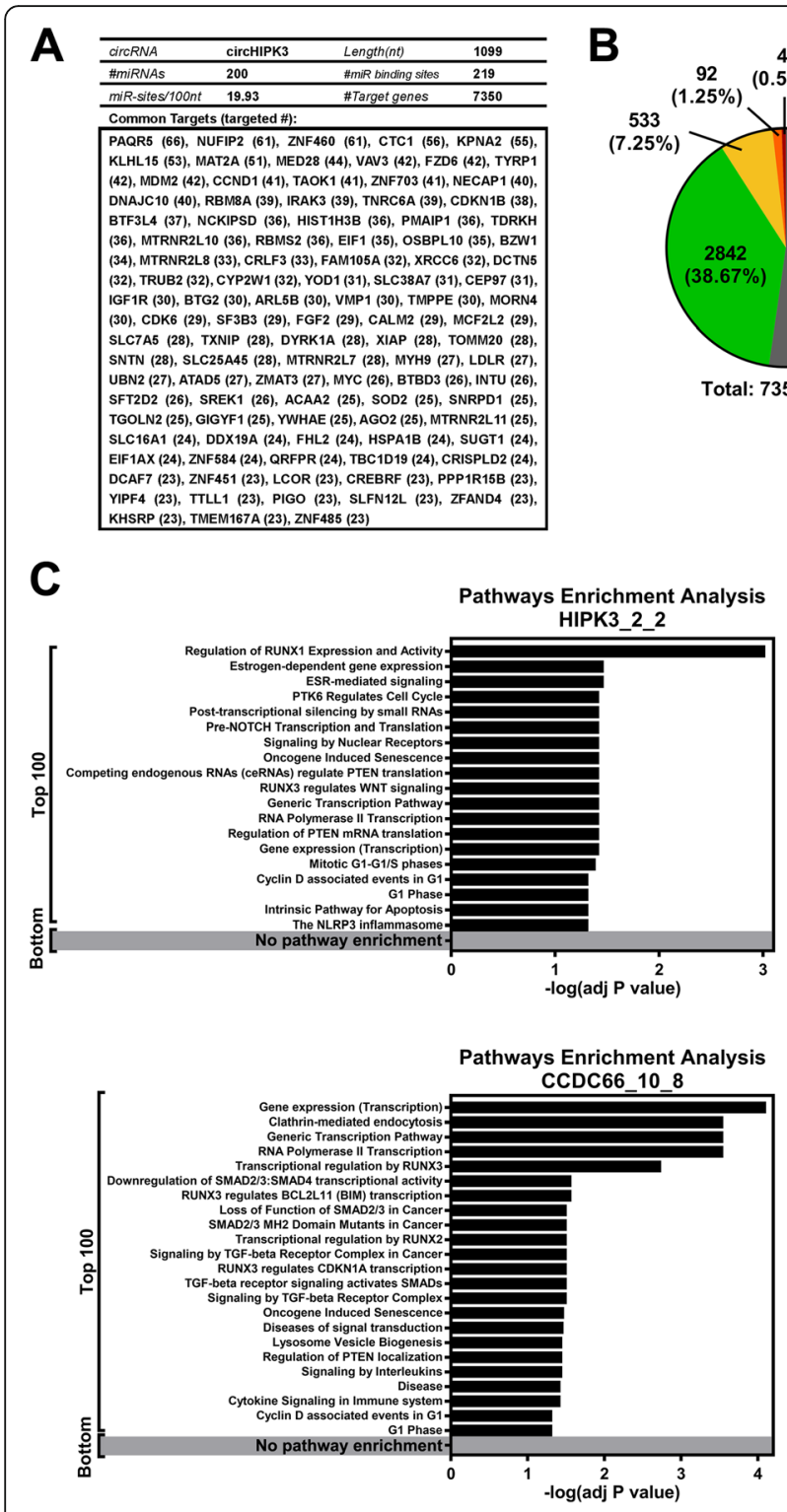

B

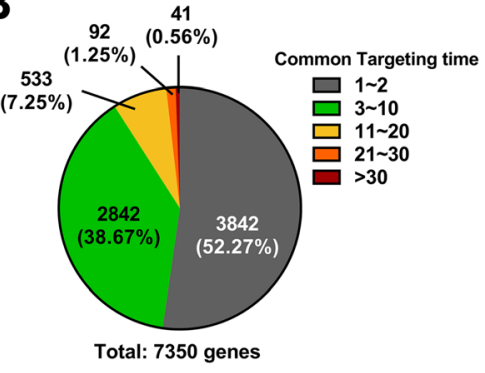

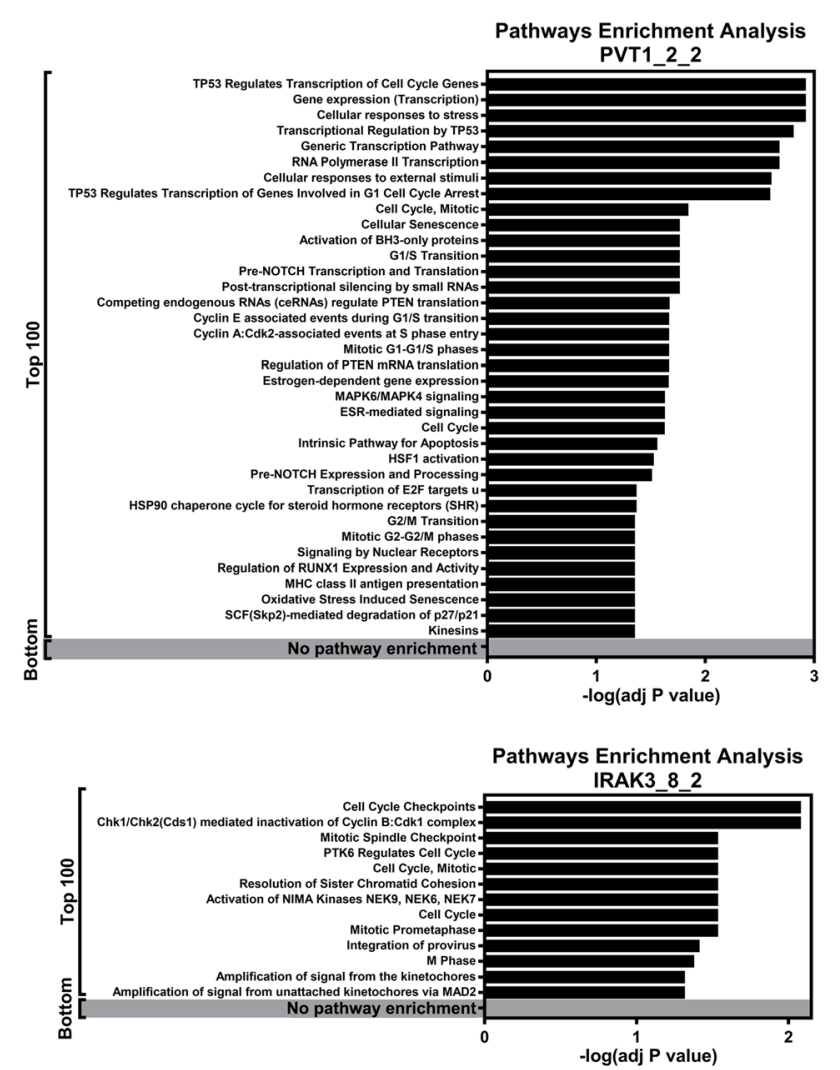

Fig. 3 ACT-selected genes identified important biological pathways. a The exported ACT results for circHIPK3. The top 100 genes ranked by their common targeting times (parenthesized) are shown at the bottom. $\mathbf{b}$ The distribution of the common targeting times of circHIPK3-regulated candidate genes is shown as a pie chart. c The ACT-prioritized genes (top 100) and low ranked genes (bottom) from circHIPK3, circCCDC66, circPVT1 and circlRAK3 were subjected to pathway enrichment analysis

pairing score or binding energy in the given circRNA sequence were subjected to pathway enrichment analysis. While enrichment analyses from the gene lists derived from the ranked energy or scores failed to distinguish circRNAs with/without sponge activity from multiple miRNAs (Fig. 4, left and central panels), pathway analyses with ACT-selected gene lists showed significantly more convergent pathways (Fig. 4, right panel). This implied that the genes targeted multiple times by circRNA-associated miRNAs tend to be more biologically significant. ACT is a novel tool to dissect the molecular and cellular functions of circRNAs. Compared to other pioneer databases for annotating circRNA/miRNA interactions (Additional file 1: Table S3) $[13,14,27]$, ACT not only provides a list of interacting miRNAs, but also a ranked gene list in a manageable length ready for further functional and/or experimental characterization. 


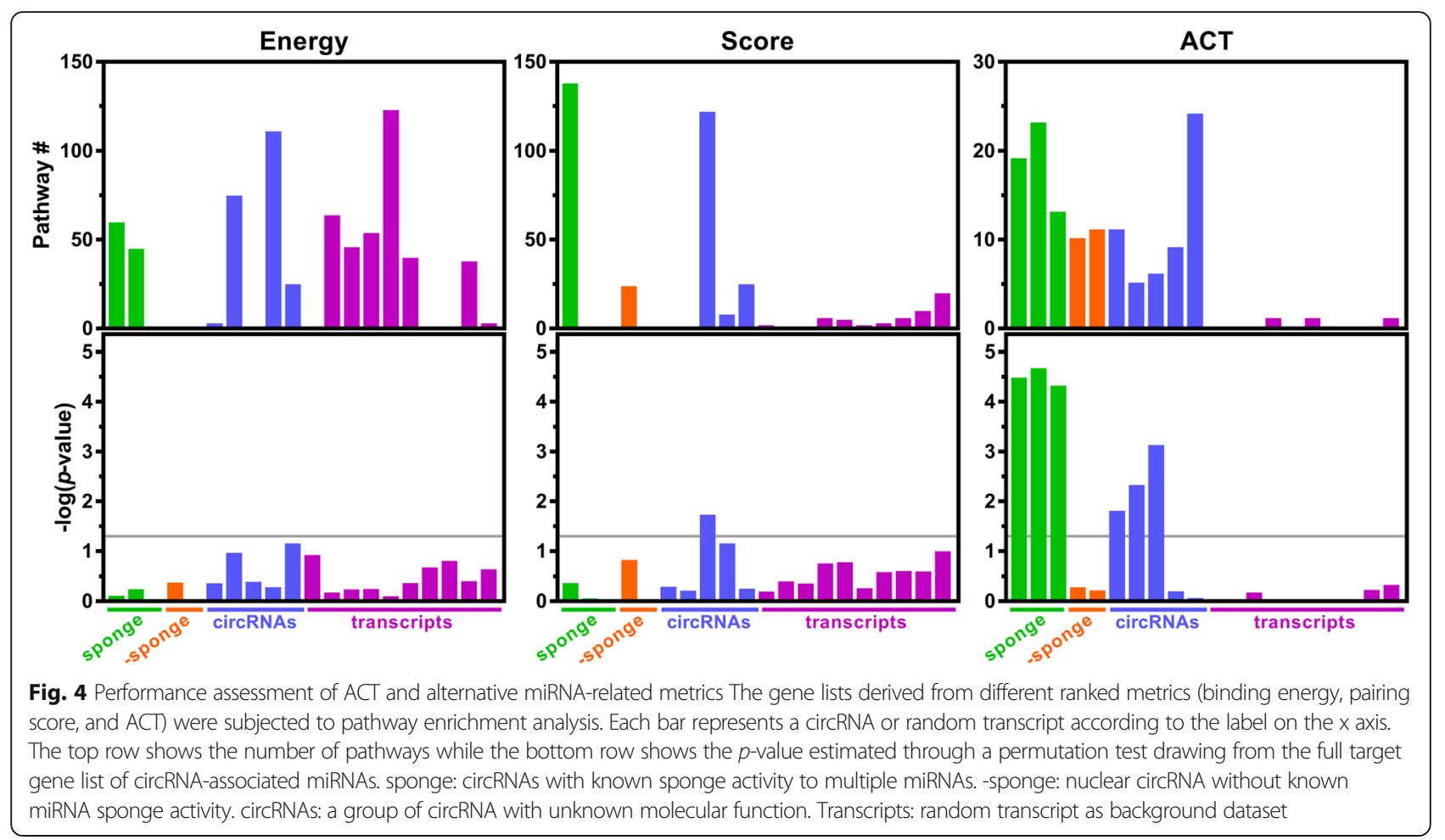

\section{Conclusion}

Taken together, analysis using ACT-selected genes provided a novel and intuitive method to differentiate the molecular and biological functions of circRNAs. Incorporating the concept of co-regulation by multiple circRNA-associated miRNAs provides a straightforward method for assessing the potential targets of circRNAs and will help prioritize the candidates as well as identify major pathways for the functional study of circRNAs.

\section{Availability and requirements \\ Project name: ACT}

Project home page: http://lab-x-omics.nchu.edu.tw/ ACT_Server/

Operating system(s): Platform independent (Webbased service)

Programming language: Perl 5 and R 3.4.3

Other requirements: N/A

License: GNU GPL; non-academic user: license needed

\section{Additional file}

Additional file 1: Figure S1. A schematic illustration of miRNA-gene interaction. Figure S2. Metrics comparison for circRNA-associated miRNAs. Table S1. The miRNA-related metrics for circRNA. Table S2. Gene lists from ACT for pathway analysis. Table S3. The comparison of platforms/ tools for circRNA-miRNA-gene network. (PDF $270 \mathrm{~kb}$ )

\section{Abbreviations}

ACT: Analysis of common targets; circRNA: Circular RNA; CT: Common targeting time; miRNA: MicroRNA

\section{Acknowledgements}

We deeply appreciate the full support of the Bioinformatics Center at National Cheng Kung University and also Mr. Yu-Cheng Chen for his technical support with establishing the website.

\section{Authors' contributions}

YCLi, YCLe and KLC developed the concept and algorithm. YCLi and KYH integrated the databases, implemented the codes and established the web service. YCLi, YCLe and KLC analyzed and evaluated the results. YCLi and YCLe drafted the manuscript. KYH revised the manuscript and supervised the project. All authors have read and approved the final version of the manuscript for publication.

\section{Funding}

Startup funds from the Institute of Biochemistry, College of Life Sciences, and National Chung Hsing University (10717073G) to KY Hsiao. Hsing Chung inter-institutional project (NCHU-CSMU-10703) to KY Hsiao and YC Lee. Ministry of Science and Technology of Taiwan (MOST 107-2320-B-005-002-MY2) to KY Hsiao. None of the funding agencies were involved in the design of the study, analysis, interpretation of data or in writing the manuscript.

Availability of data and materials

Web service is made freely available at http://lab-x-omics.nchu.edu.tw/ACT_ Server.

Ethics approval and consent to participate

Not applicable.

\section{Consent for publication}

Not applicable.

Competing interests

The authors declare that they have no competing interests. 


\section{Author details}

'Department of Plant Pathology, College of Agriculture and Natural Resources, National Chung Hsing University, Taichung 40227, Taiwan. ${ }^{2}$ Department of Biotechnology, Asia University, Taichung 41354, Taiwan. ${ }^{3}$ Department of Radiation Oncology, Chung Shan Medical University Hospital, Taichung 40201, Taiwan. ${ }^{4}$ School of Medicine, Chung Shan Medical University, Taichung 40201, Taiwan. ${ }^{5}$ Department of Physiology, National Cheng Kung University, Tainan 70101, Taiwan. ${ }^{6}$ Institute of Biochemistry, College of Life Sciences, National Chung Hsing University, Taichung 40227, Taiwan. ${ }^{7}$ Program in Translational Medicine, College of Life Sciences, National Chung Hsing University, Taichung 40227, Taiwan. ${ }^{8}$ Rong Hsing Research Center for Translational Medicine, College of Life Sciences, National Chung Hsing University, Taichung 40227, Taiwan. 'Bachelor Program of Biotechnology, College of Agriculture and Natural Resources, National Chung Hsing University, Taichung 40227, Taiwan.

\section{Received: 13 March 2019 Accepted: 24 June 2019}

Published online: 02 July 2019

\section{References}

1. Salzman J, Gawad C, Wang PL, Lacayo N, Brown PO. Circular RNAs are the predominant transcript isoform from hundreds of human genes in diverse cell types. PLoS One. 2012;7(2):e30733.

2. Memczak S, Jens M, Elefsinioti A, Torti F, Krueger J, Rybak A, Maier L, Mackowiak SD, Gregersen LH, Munschauer M, et al. Circular RNAs are a large class of animal RNAs with regulatory potency. Nature. 2013;495(7441):333-8.

3. Guo JU, Agarwal V, Guo H, Bartel DP. Expanded identification and characterization of mammalian circular RNAs. Genome Biol. 2014;15(7):409.

4. Legnini I, Di Timoteo G, Rossi F, Morlando M, Briganti F, Sthandier O, Fatica A, Santini T, Andronache A, Wade M, et al. Circ-ZNF609 is a circular RNA that can be translated and functions in Myogenesis. Mol Cell. 2017;66(1):2237 e29.

5. Li Z, Huang C, Bao C, Chen L, Lin M, Wang X, Zhong G, Yu B, Hu W, Dai L, et al. Exon-intron circular RNAs regulate transcription in the nucleus. Nat Struct Mol Biol. 2015;22(3):256-64

6. Holdt LM, Stahringer A, Sass K, Pichler G, Kulak NA, Wilfert W, Kohlmaier A, Herbst A, Northoff BH, Nicolaou A, et al. Circular non-coding RNA ANRIL modulates ribosomal RNA maturation and atherosclerosis in humans. Nat Commun. 2016;7:12429.

7. Du WW, Yang W, Liu E, Yang Z, Dhaliwal P, Yang BB. Foxo3 circular RNA retards cell cycle progression via forming ternary complexes with p21 and CDK2. Nucleic Acids Res. 2016;44(6):2846-58.

8. Hsiao KY, Sun HS, Tsai SJ. Circular RNA - new member of noncoding RNA with novel functions. Exp Biol Med (Maywood). 2017:242(11):1136-41.

9. Hansen TB, Jensen TI, Clausen BH, Bramsen JB, Finsen B, Damgaard CK, Kjems J. Natural RNA circles function as efficient microRNA sponges. Nature. 2013:495(7441):384-8.

10. Zheng Q, Bao C, Guo W, Li S, Chen J, Chen B, Luo Y, Lyu D, Li Y, Shi G, et al. Circular RNA profiling reveals an abundant circHIPK3 that regulates cell growth by sponging multiple miRNAs. Nat Commun. 2016;7:11215.

11. Hsiao KY, Lin YC, Gupta SK, Chang N, Yen L, Sun HS, Tsai SJ. Noncoding effects of circular RNA CCDC66 promote Colon Cancer growth and metastasis. Cancer Res. 2017;77(9):2339-50.

12. Liu YC, Hong HC, Yang CD, Lee $W H$, Huang HT, Huang HD. Ouroboros resembling competitive endogenous loop (ORCEL) in circular RNAs revealed through transcriptome sequencing dataset analysis. BMC Genomics. 2018; 19(Suppl 2):171.

13. Liu YC, Li JR, Sun CH, Andrews E, Chao RF, Lin FM, Weng SL, Hsu SD, Huang CC, Cheng C, et al. CircNet: a database of circular RNAs derived from transcriptome sequencing data. Nucleic Acids Res. 2016;44(D1):D209-15.

14. Li JH, Liu S, Zhou H, Qu LH, Yang JH. starBase v2.0: decoding miRNA-ceRNA, miRNA-ncRNA and protein-RNA interaction networks from large-scale CLIPSeq data. Nucleic Acids Res. 2014;42(Database issue):D92-7.

15. Ghosal S, Das S, Sen R, Basak P, Chakrabarti J. Circ2Traits: a comprehensive database for circular RNA potentially associated with disease and traits. Front Genet. 2013:4:283.

16. Zheng J, Liu X, Xue Y, Gong W, Ma J, Xi Z, Que Z, Liu Y. TTBK2 circular RNA promotes glioma malignancy by regulating miR-217/HNF1beta/Derlin-1 pathway. J Hematol Oncol. 2017;10(1):52.

17. Zhang J, Liu H, Hou L, Wang G, Zhang R, Huang Y, Chen X, Zhu J. Circular RNA_LARP4 inhibits cell proliferation and invasion of gastric cancer by sponging miR-424-5p and regulating LATS1 expression. Mol Cancer. 2017; 16(1):151.

18. Chen G, Shi Y, Liu M, Sun J. circHIPK3 regulates cell proliferation and migration by sponging miR-124 and regulating AQP3 expression in hepatocellular carcinoma. Cell Death Dis. 2018;9(2):175.

19. Kozomara A, Griffiths-Jones S. miRBase: annotating high confidence microRNAs using deep sequencing data. Nucleic Acids Res. 2014; 42(Database issue):D68-73.

20. Griffiths-Jones S. The microRNA registry. Nucleic Acids Res. 2004;32(Database issue):D109-11.

21. Enright AJ, John B, Gaul U, Tuschl T, Sander C, Marks DS. MicroRNA targets in drosophila. Genome Biol. 2003;5(1):R1.

22. Chien $\mathrm{CH}$, Sun YM, Chang WC, Chiang-Hsieh PY, Lee TY, Tsai WC, Horng JT, Tsou AP, Huang HD. Identifying transcriptional start sites of human microRNAs based on high-throughput sequencing data. Nucleic Acids Res. 2011;39(21):9345-56

23. Panda AC, Grammatikakis I, Kim KM, De S, Martindale JL, Munk R, Yang X, Abdelmohsen K, Gorospe M. Identification of senescence-associated circular RNAs (SAC-RNAs) reveals senescence suppressor CircPVT1. Nucleic Acids Res. 2017:45(7):4021-35.

24. Wu J, Jiang Z, Chen C, Hu Q, Fu Z, Chen J, Wang Z, Wang Q, Li A, Marks JR, et al. CirclRAK3 sponges miR-3607 to facilitate breast cancer metastasis. Cancer Lett. 2018;430:179-92.

25. Chen N, Zhao G, Yan X, Lv Z, Yin H, Zhang S, Song W, Li X, Li L, Du Z, et al. A novel FLI1 exonic circular RNA promotes metastasis in breast cancer by coordinately regulating TET1 and DNMT1. Genome Biol. 2018;19(1):218.

26. Qin M, Wei G, Sun X. Circ-UBR5: an exonic circular RNA and novel small nuclear RNA involved in RNA splicing. Biochem Biophys Res Commun. 2018; 503(2):1027-34.

27. Xia S, Feng J, Chen K, Ma Y, Gong J, Cai F, Jin Y, Gao Y, Xia L, Chang H, et al. CSCD: a database for cancer-specific circular RNAs. Nucleic Acids Res. 2018; 46(D1):D925-9.

\section{Publisher's Note}

Springer Nature remains neutral with regard to jurisdictional claims in published maps and institutional affiliations.

Ready to submit your research? Choose BMC and benefit from:

- fast, convenient online submission

- thorough peer review by experienced researchers in your field

- rapid publication on acceptance

- support for research data, including large and complex data types

- gold Open Access which fosters wider collaboration and increased citations

- maximum visibility for your research: over $100 \mathrm{M}$ website views per year

At $\mathrm{BMC}$, research is always in progress.

Learn more biomedcentral.com/submissions 